\title{
Accreditation and attribution in data sharing
}

\section{To the Editor:}

In the editorial in your June issue 'Credit where credit is overdue ${ }^{1}$ discussing obstacles to sharing of research data, you highlight accreditation to researchers who deposit their data in public archives as an important incentive strategy. Treating data as publications and leveraging peer recognition to motivate data sharing is key to tackling numerous issues surrounding research data availability ${ }^{2,3}$. As you point out, digital object identifiers (DOIs) can be recruited to identify published datasets. Such an approach has been piloted for several years in a limited number of scientific domains and efforts are now ongoing to widen the scope of 'data DOls' to other kinds of research data. But a number of long- and short-term obstacles will have to be overcome if datasets are to become first-class, citable citizens of the scientific literature.

DOls and other persistent identifier schemes address the nagging problem of Web hyperlink decay or 'link rot' in the literature, resulting from citations to Internet references ${ }^{4}$. In the scholarly and professional publishing domain, CrossRef (http://www.crossref.org), a not-for-profit association comprising over 700 publishers, libraries and other stakeholders, provides technological and social infrastructure which supports identifying, locating and citing publications via DOls.

Although efforts to leverage the benefits of DOls as persistent identifiers of datasets published online has not yet gained much traction in the biological sciences, they have been ongoing in other disciplines for some time. In collaboration with several international data centers in the Earth and Environmental Sciences, The German National Library of Science and Technology (TIB, http://www.tib-hannover.de) has been registering DOls for large-scale primary datasets since $2005^{5,6}$. In this system, TIB serves the same purpose as CrossRef and maintains a central catalog of DOls and corresponding publisher-provided metadata, or data describing each dataset (e.g. title, authors and date published). The metadata also include a Uniform Resource Locator (URL) link to the data centre where the dataset can be accessed. An example of a data DOI is 10.1594/PANGAEA.119754, which identifies the dataset cited in ref $^{7}$. Following the link, or resolving the DOI, via the central DOI resolver (http://dx.doi.org) in a Web browser takes the user to the PANGEA openaccess library for geoscientific and environmental data (http://www.pangaea.de) where the dataset is archived.

An important feature of data DOls is that a published dataset has a separate and unique identity of its own and can be cited directly and unambiguously, instead of indirectly via a parent journal publication. Citation data can be aggregated and combined with web access data to systematically credit dataset creators for their contributions ${ }^{8}$, and used to calculate a variety of per-dataset citation metrics ${ }^{9}$. Such methods may be far more appropriate for assessing the importance of scientific contributions than the controversial Impact Factor ${ }^{10}$. Furthermore, the system facilitates data publishing without a traditional journal publication. This has implications on several fronts, not the least as a means to address problems stemming from 'publication bias' in fields such as genetic epidemiology, whereby negative, 'uninteresting' findings are much less frequently published in journals than positive findings ${ }^{11,12}$. One can easily envision a biomedical data registration and archiving service operating in similar fashion to the PLoS ONE open-access journal (http://www.plosone.org), offering a platform for investigators to publish peer-reviewed 
data from scientifically sound research which would otherwise not be published.

The TIB registry holds DOIs and metadata on $\sim 600,000$ primary datasets from geoscientific, climate and environmental research. Data DOls have therefore been largely limited to those fields, but this is now changing. Following the signing of a Memorandum of Understanding earlier this year, TIB has joined forces with several international partners to form the DataCite initiative (http://www.datacite.org). This consortium includes national research libraries and information centers in Germany, France, the Netherlands, Switzerland, Denmark, United Kingdom and Canada, with a common goal of establishing a worldwide data registration agency and promote the use of DOls for scientific data. Hopefully the next few years will see major biological data centers like EBI/EMBL and $\mathrm{NCBI}$ joining this initiative.

As important as the above developments may prove, it is important to note that 'tagging' published datasets with DOls is not a magic bullet and numerous other factors need to be taken into account. First, making datasets citable does not in itself ensure that authors actually cite them appropriately, so normal rules of scientific etiquette apply when it comes to crediting data contributions from others. Second, to ensure persistence, databases for archiving primary data and keeping them accessible must be developed (if none exist in a given domain), and sustainable, long-term funding ensured for such critical research infrastructure ${ }^{13,14}$. Third, shared data does not necessarily equate reusable, useful data ${ }^{15,16}$, so in order to enhance future reuse value further emphasis needs to be placed on the use of community data standards, as well as recognition and career advancement options for biocurators who manage and curate the data ${ }^{17}$. Last, but not least, it is critical that issues relating to identity of researchers be considered in data registration schemes. Recent developments to create unique, persistent identifiers for authors and contributors in general are steps in the right direction ${ }^{18-20}$. In particular, a centralized system for assigning and managing such contributor IDs, currently being developed by CrossRef in collaboration with the major publishers ${ }^{21}$, could be integrated directly with data registration systems. This would facilitate unambiguous linking of IDs for investigators with DOls for datasets they contribute to.

Recent publications in a special issue of your sister journal Nature ${ }^{22}$ and elsewehere ${ }^{23}$ highlight the complexity of the data sharing problem in molecular biology, especially in this age of 'big data' ${ }^{24}$. The situation can be likened to a jigzaw puzzle with many interlocking pieces. Providing a means for systematically rewarding researchers for the extra effort to publish their data would put an important piece of that puzzle in the right place, and help to complete the picture. The fact that this might be accomplished by extending and adapting existing infrastructure for traditional publication means that the technological hurdles should be minimal. In fact, the challenges to wide adoption of data DOls may prove to be of a non-technical nature: operational overheads (i.e. yet another identifier system to maintain) and the cost of registering large numbers of DOls may be significant barriers for many data centers on limited budgets. Nevertheless, if these challenges can be met and we can start tracking data contributions, data citations and data access globally and in a fine-grained way, a major obstacle to data sharing will have been greatly alleviated.

Gudmundur A. Thorisson 
Department of Genetics, University of Leicester, United Kingdom

e-mail: gt50@leicester.ac.uk

1. Editors. Credit where credit is overdue. Nature Biotechnology 27, 579 (2009). doi:10.1038/nbt0709-579

2. Costello, M. Motivating Online Publication of Data. BioScience 59, 418-427 (2009). doi:10.1525/bio.2009.59.5.9

3. Smith, V. Data publication: towards a database of everything. BMC Res Notes 2, 113 (2009). doi:10.1186/1756-0500-2-113

4. Dellavalle, R. et al. Information science. Going, going, gone: lost Internet references. Science 302, 787-8 (2003). doi:10.1126/science.1088234

5. Brase, J. Using Digital Library Techniques - Registration of Scientific Primary Data. Research and Advanced Technology for Digital Libraries 488-494 (2004). doi:10.1007/b100389

6. Brase, J. \& Schindler, U. The publication of scientific data by World Data Centers and the National Library of Science and Technology in Germany. Data Science Journal 5, 205-208 (2006). doi:10.2481/dsj.5.205

7. Stein, R. \& Fahl, K. Distribution of grain size and clay minerals in surface sediments of the Kara Sea. PANGEA (2003). doi:10.1594/PANGAEA.119754

8. Editors. Compete, collaborate, compel. Nature Genetics 39, 931 (2007). doi:10.1038/ng0807-931

9. Bollen, J. et al. A principal component analysis of 39 scientific impact measures. PLoS ONE 4, e6022 (2009). doi:10.1371/journal.pone.0006022

10. Campbell, P. Escape from the impact factor. Ethics Sci. Environm. Polit. 8, 5-7 (2008). doi:10.3354/esep00078

11. Brookes, A. \& Prince, J. Genetic association analysis: lessons from the study of Alzheimers disease. Mutat Res 573, 152-9 (2005). doi:10.1016/j.mrfmmm.2004.08.017

12. Shields, P. Publication bias is a scientific problem with adverse ethical outcomes: the case for a section for null results. Cancer Epidemiol Biomarkers Prev 9, 771-772 (2000).

13. Ball, C., Sherlock, G. \& Brazma, A. Funding high-throughput data sharing. Nature Biotechnology 22, 1179-83 (2004). doi:10.1038/nbt0904-1179

14. Merali, Z. \& Giles, J. Databases in peril. Nature 435, 1010-1011 (2005). doi:10.1038/4351010a 
15. Ioannidis, J. et al. Repeatability of published microarray gene expression analyses. Nature Genetics 41, 149-55 (2009). doi:10.1038/ng.295

16. Editors Mostly, your results matter to others. Nature Genetics 41, 135 (2009). doi:10.1038/ng0209-135

17. Howe, D. et al. Big data: The future of biocuration. Nature 455, 47-50 (2008). doi:10.1038/455047a

18. Bourne, P.E. \& Fink, J.L. I am not a scientist, I am a number. PLoS Comput Biol 4, e1000247 (2008). doi:10.1371/journal.pcbi.1000247

19. Cals, J.W.L. \& Kotz, D. Researcher identification: the right needle in the haystack. Lancet 371, 2152-3 (2008). doi:10.1016/S0140-6736(08)60931-9

20. Qiu, J. Scientific publishing: Identity crisis. Nature News 451, 766 (2008). doi:10.1038/451766a

21. Enserink, M. Scientific publishing. Are you ready to become a number? Science 323, 1662-4 (2009). doi:10.1126/science.323.5922.1662

22. Data sharing special issue. Data's shameful neglect. Nature 461, 145 (2009). doi:10.1038/461145a

23. Field, D. et al. Megascience. 'Omics data sharing. Science 326, 234-6 (2009). doi:10.1126/science.1180598

24. Big Data special issue. Community cleverness required. Nature 455, 1 (2008). doi:10.1038/455001a 